\title{
Association Between Metabolic Syndrome and Its Components with Severity of Acute Pancreatitis
}

This article was published in the following Dove Press journal:

Diabetes, Metabolic Syndrome and Obesity: Targets and Therapy

\author{
Ramin Niknam (D) \\ Jamshid Moradi ${ }^{1}$ \\ Keramat Allah Jahanshahi ${ }^{2}$ \\ Laleh Mahmoudi ${ }^{3}$ \\ Fardad Ejtehadi $\mathbb{1}^{1}$ \\ 'Gastroenterohepatology Research \\ Center, Shiraz University of Medical \\ Sciences, Shiraz, Iran; ${ }^{2}$ Faculty of Medical \\ School, Yasuj University of Medical \\ Sciences, Yasuj, Iran; ${ }^{3}$ Department of \\ Clinical Pharmacy, School of Pharmacy, \\ Shiraz University of Medical Sciences, \\ Shiraz, Iran
}

Correspondence: Fardad Ejtehadi Gastroenterohepatology Research Center, Shiraz University of Medical Sciences, Shiraz, Iran

$\mathrm{Tel}+98917|1| 3439 \mid$

$\mathrm{Fax}+987136281442$

Email edjtehadif@sums.ac.ir
Background: Acute pancreatitis (AP) and metabolic syndrome (MetS) are common clinical problems with serious complications and high cost of treatment. The relationship between MetS and severity of AP is unknown, and because there are very few published articles, further studies had been recommended. Therefore, we designed this study to estimate the association between MetS and its components with AP severity.

Patients and Methods: In this prospective analytical cross-sectional study, all AP patients who referred to two referral hospitals affiliated with Shiraz University of Medical Sciences between 2016 and 2019 were evaluated. The diagnosis of MetS syndrome as well as follow-up of patients for evaluation of severity and outcome of AP was performed in all enrolled patients. National Cholesterol Education Program Adult Treatment Panel III (NCEP/ATP-III) was used for diagnosis of MetS. Bedside Index for Severity in Acute Pancreatitis (BISAP) and revised Atlanta classification (RAC) were used to estimate the severity of AP. Finally, the association between MetS syndrome and its components at different AP severities was compared.

Results: Of the 214 patients referred to our center, a total of 76 patients with AP were included in this study. Of the included patients, $40(52.6 \%)$ were male and $36(47.4 \%)$ were female. Frequency of patients in Mets and non-MetS groups was 34 (44.74\%) and 42 (55.26\%), respectively. The gender and mean age difference between Mets and non-MetS groups were not statistically significant. According to Mets groups, 25 (73.5\%) and 9 (26.5\%) AP patients with MetS had BISAP score of $0-2$ and 3-5, respectively. BISAP score in MetS group was significantly higher than non-MetS group $(\mathrm{P}=0.022)$. Overall, $20(58.8 \%), 11(32.4 \%)$, and $3(8.8 \%)$ of AP patients with MetS had mild, moderately severe, and severe AP based on RAC, respectively. Severe RAC was significantly higher in the MetS group than in the non-MetS group $(\mathrm{P}=0.029)$. The frequency of complications in MetS group was significantly higher than in non-MetS group ( $\mathrm{P}=0.008)$. The length of hospital stay was also significantly higher in the MetS group than in the non-MetS group $(\mathrm{P}=0.001)$. Overall, three patients died, two of whom had MetS and all had severe RAC and BISAP 3-5. Waist circumference, fasting blood sugar, and triglyceride were significantly higher in the severe and moderately severe types of AP group than in the mild AP group, according to RAC. Although, mean of all components of MetS except HDL-cholesterol were higher in BISAP of 3-5 group than BISAP of 0-2 group, the difference was only significant in waist circumference.

Conclusion: The present study showed that there was a significant association between the rate of MetS and its components including waist circumference, hyperglycemia and hypertriglyceridemia with increasing AP severity. This study showed that the incidence of more severe AP in patients with MetS is much higher than in patients without MetS. We recommend that the new studies with larger sample sizes are conducted with careful consideration of confounding factors to investigate the relationship between the severity of MetS and AP as well as the effect of MetS treatment on the nature of AP.

Keywords: acute pancreatitis, metabolic syndrome, Bedside Index of Severity in Acute Pancreatitis, BISAP, revised Atlanta classification, severity

Diabetes, Metabolic Syndrome and Obesity: Targets and Therapy 2020:13 1289-1296

1289

DovePress if in $>$

(c) (i) $\odot 2020$ Niknam et al. This work is published and licensed by Dove Medical Press Limited. The full terms of this license are available at https://www.dovepress.com/terms. (C) work you hereby accept the Terms. Non-commercial uses of the work are permitted without any further permission from Dove Medical Press Limited, provided the work is properly attributed. For permission for commercial use of this work, please see paragraphs 4.2 and 5 of our Terms (https://www.dovepress.com/terms.php). 


\section{Introduction}

Acute pancreatitis (AP) is a common clinical problem with high costs of treatment caused by pancreatic inflammation and can lead to complications or multi-organ involvement. AP is defined by severe abdominal pain, elevated serum lipase or amylase, and characteristic AP findings in imaging. The most common causes of AP in adults are alcohol and gallstones. Most cases of acute pancreatitis are mild and self-limiting, but severe AP has high mortality and early detection is particularly important for proper management. ${ }^{1,2}$

Different types of severity scoring systems have been developed to detect severe AP. The Bedside Index for Severity in Acute Pancreatitis (BISAP) is a simple scoring system that predicts the severity of disease. ${ }^{3-6}$ Revised Atlanta classification (RAC) is another clinical and radiological classification to estimate the severity of AP that organ failure and local or systemic complications are used for this type of classification. ${ }^{7-9}$

Metabolic syndrome (MetS) as a common problem is the combination of metabolic abnormalities including central obesity, hyperglycemia, hypertriglyceridemia, highdensity lipoprotein cholesterol (HDL-C), and hypertension. MetS is associated with increased mortality and morbidity but lifestyle modifications and some medication can reverse metabolic risk factors. ${ }^{10,11}$

Most studies have suggested that obesity has a negative effect on the AP process, but relationship between MetS and severity of AP is unclear, and there is a paucity of literature about this issue. ${ }^{12,13}$ On the other hand, the high prevalence and cost of MetS and AP provides important conditions in which further studies are needed. ${ }^{12,14,15}$ Therefore, we designed this study to estimate the association between MetS and its components with severity of AP in southern Iran.

\section{Patients and Methods}

\section{Participants}

A prospective observational study was performed at two referral hospitals affiliated with Shiraz University of Medical Sciences between 2016 and 2019. Patients presenting with AP were admitted to these hospitals and were thoroughly evaluated. Exclusion criteria as conditions that could act as confounders for our variables were the history of recurrence of AP, other pancreatic disorders (eg chronic pancreatitis, pancreatic mass or cyst, and previous pancreatic surgery or trauma), pregnancy, known case of malignancy, illicit drug abusers, and non-cooperative patients. All eligible patients were followed for severity and outcome of AP.

Diagnosis of AP and MetS syndrome as well as followup of patients and evaluation of AP severity was performed by a gastroenterologist for three months. Various necessary variables including sex, age, weight, height, laboratory data were collected and recorded in the checklist. Finally, the association between MetS syndrome and its components at different AP severities was compared. The primary endpoints were association between the presence of MetS and severity of AP, complications of AP, length of hospital stay, and mortality. Secondary endpoints were association between metabolic syndrome components and severity of AP.

\section{Ethical Approval/Statement}

The study was conducted after the Helsinki Ethics Declaration in Medical Research and approved by the Ethics Committee of Shiraz University of Medical Sciences (95-01-01-11662). Written informed consent was obtained from all patients for medical examination before starting the study.

\section{Definitions}

AP was defined as presence 2 of the following three criteria: abdominal pain suggestive of AP; serum lipase or amylase levels 3 or more times normal; and characteristic findings of AP on imaging (trans-abdominal ultrasound, computed tomography, or magnetic resonance). ${ }^{1,2}$

National Cholesterol Education Program Adult Treatment Panel III (NCEP/ATP-III) was used for diagnosis of MetS. ${ }^{11,16}$ Metabolic syndrome was defined according to NCEP/ATP-III criteria by the presence of at least three of the following metabolic abnormalities: waist circumference $>102$ and $>88 \mathrm{~cm}$ in men and women, triglyceride $\geq 150 \mathrm{mg} / \mathrm{dL}$, high-density lipoprotein cholesterol (HDL-C) $<40$ and $<50 \mathrm{mg} / \mathrm{dL}$ in men and women, blood pressure $\geq 130 / 85 \mathrm{mmHg}$; and fasting blood sugar $\geq 100 \mathrm{mg} / \mathrm{dL}$.

\section{Severity of Acute Pancreatitis}

BISAP score ${ }^{4}$ and RAC $^{2,7,8}$ were calculated from data collected to define severe AP. Components of BISAP scoring system were included blood urea nitrogen $>25 \mathrm{mg} / \mathrm{dl}$, impaired mental status, systemic inflammatory response syndrome, Age $>60$ years, and pleural effusion. ${ }^{3,4}$ Based on this score, our patients were classified into two groups: BISAP $0-2$ and BISAP $3-5 .{ }^{6}$ The RAC was also used to estimate the 
severity of AP. The presence of local or systemic complications and/or organ failure such as infected necrosis and peripancreatic fluid collections were used to classify AP based on this classification. Our patients were classified into mild, moderately severe, and severe AP according to RAC, respectively. Mild AP was defined as having no organ failure and local or systemic complications. Moderately severe AP was defined as having transient organ failure ( $<48$ hours) and/or local or systemic complications. Severe AP was defined as having persistent organ failure for more than 48 hours. ${ }^{2,7-9}$

\section{Measurements}

Laboratory tests, ultrasound scan of the abdomen and pelvis, and plain radiograph of the abdomen and chest were performed on admission. Laboratory blood tests included amylase, lipase, hematocrit, leukocyte count, glucose level, creatinine, urea, calcium, electrolytes, lipid profile test, and arterial blood gas analysis. Abdominopelvic CT scan was done in complicated patients.

Waist circumference between the lowest rib and iliac crest was measured at level of umbilicus in centimeter. Systolic and diastolic blood pressure was measured on the left upper arm using a random-zero sphygmomanometer. All laboratory parameters are examined in a specialized laboratory. Blood samples were transferred to the laboratory in less than an hour in ice. Serum glucose was measured using glucose-peroxidase colorimetric enzymatic method with sensitivity of $5 \mathrm{mg} / \mathrm{dL}$ and intra-assay coefficients of variation (CV) of $1.4 \%$ in upper limit and $1.7 \%$ in lower limit concentrations. The Inter-assay $\mathrm{CV}$ for the assay was $0.6 \%$ in upper limit and $1.1 \%$ in lower limit concentrations. Serum cholesterol was measured with colorimetric method with a sensitivity of $5 \mathrm{mg} / \mathrm{dl}$. The Intraassay and inter-assay CV for the assay was $1.6 \%$ in lower limit and $0.6 \%$ for upper limit concentrations. Serum triglyceride was measured with colorimetric method with a sensitivity of $5 \mathrm{mg} / \mathrm{dl}$. The Intra-assay and inter-assay $\mathrm{CV}$ for the assay was $1.1 \%$ in lower limit and $0.9 \%$ for upper limit concentrations. HDL-C was measured after deposition of apolipoprotein B containing lipoproteins with phosphotungstic acid.

\section{Statistical Analysis}

The data were stored in a SPSS database and statistical analyses were performed SPSS software, version 25.0, Chicago, USA. Analysis of association between MetS and severity of AP were performed using Chi-Square test. Quantitative data were expressed as mean and standard deviation (SD) and their differences between groups were studied using two independent sample $t$ test. Where appropriate, non-parametric tests such as Mann-Whitney and Kruskal-Wallis tests were used. Prevalence ratios (PRs) and 95\% confidence intervals (CIs) were used to describe the associations and were obtained by Robust Poisson regression models. $\mathrm{P}<0.05$ was considered as significant difference.

\section{Results}

As shown in Figure 1, of the 214 evaluated patients, a total of 76 patients with definite AP were included in this study. Of the included patients, 40 (52.6\%) were male and 36 (47.4\%) were female. The mean age (SD) of the AP patients was 47.90 (15.70) ranging from 18 to 70 years. According to Mets criteria, the frequency of patients in Mets and nonMetS groups was 34 (44.74\%) and 42 (55.26\%), respectively. The mean age (SD) in Mets and non-MetS groups were 50.59 (14.07) and 45.71 (16.75), respectively, the difference between them was not statistically significant $(\mathrm{P}=0.18)$. The gender difference between Mets and non-MetS groups was also not statistically significant $(P=0.33)$. Baseline demographic and clinical characteristics of AP patients with and without Mets are shown in Table 1.

Overall, $84.2 \%$ and $15.8 \%$ patients had BISAP score of $0-2$ and 3-5, respectively. According to Mets groups, $73.5 \%$ and $26.5 \%$ AP patients with MetS had BISAP score of 0-2 and 3-5, respectively. On the other hand, $92.9 \%$ and $7.1 \%$ AP patients without MetS had BISAP score of $0-2$ and 3-5, respectively. As shown in the Table 2, BISAP score in MetS group was significantly higher than non-MetS group ( $\mathrm{P}=$ 0.022). Overall, $73.7 \%, 21.1 \%$, and $5.3 \%$ patients had mild, moderately severe, and severe AP according to RAC, respectively. $58.8 \%, 32.4 \%$, and $8.8 \%$ of AP patients with MetS had mild, moderately severe, and severe AP based on RAC, respectively. On the other hand, $85.7 \%, 11.9 \%$, and $2.4 \%$ of patients without MetS had mild, moderately severe, and severe AP, respectively. Severe AP was significantly higher in the MetS group than in the non-MetS group $(\mathrm{P}=0.029)$ (Table 2).

Systemic and local complications including fluid collection, pseudocyst, necrotizing pancreatitis, organ failure, and biliary obstruction were seen in $21(61.8 \%)$ and 10 $(23.8 \%)$ of patients with and without MetS, respectively. The frequency of complications in MetS group was significantly higher than in non-MetS group $(\mathrm{P}=0.008)$. The length of hospital stay was also significantly higher in the 


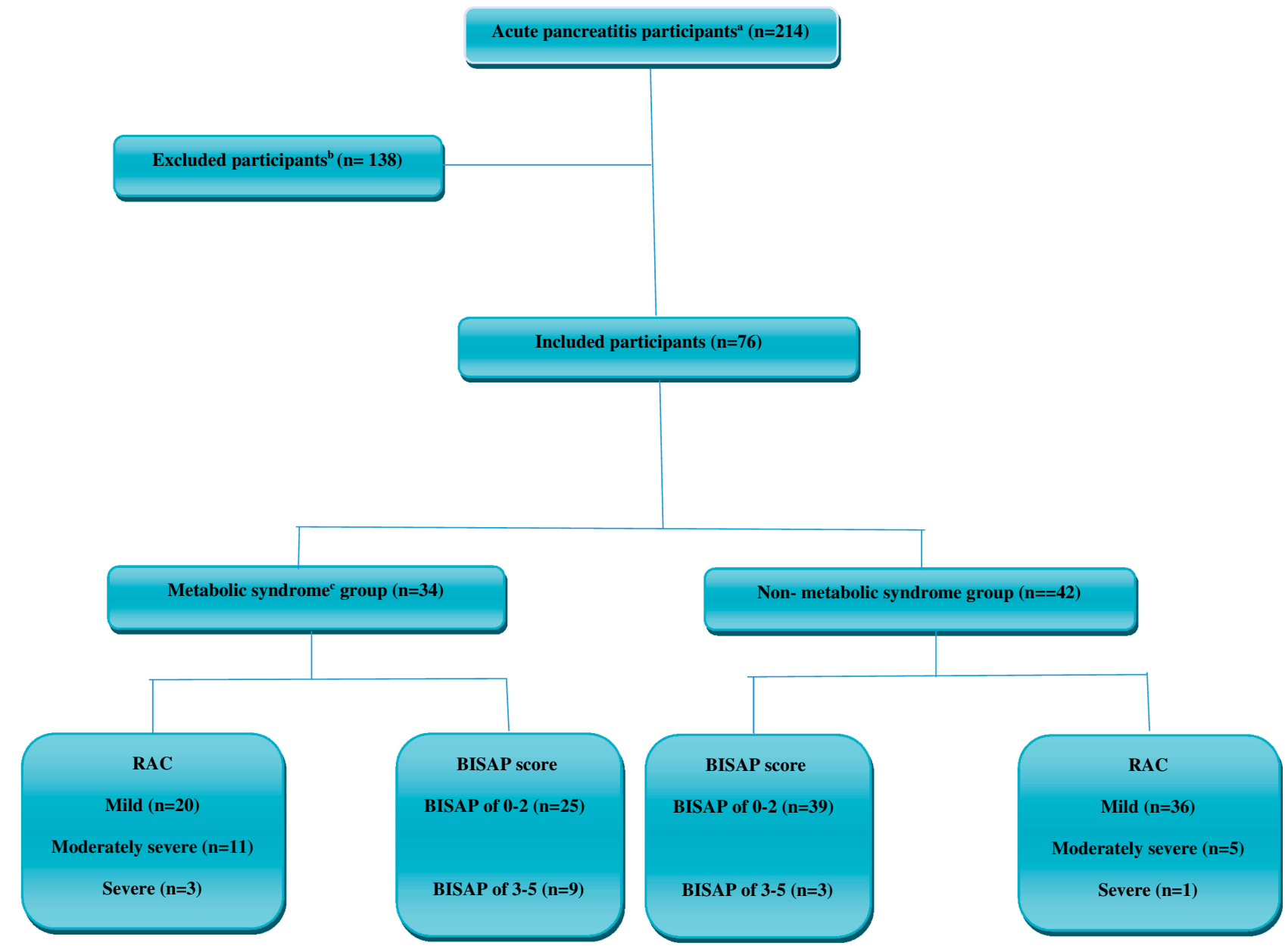

Figure I Flow diagram for the acute pancreatitis participants' selection process.

Notes: acute pancreatitis was defined as presence 2 of the following three criteria: abdominal pain suggestive of AP; serum lipase or amylase levels 3 or more times normal; and characteristic findings of AP on imaging. 'Exclusion criteria as conditions that could act as confounders for our variables were the history of recurrence of AP, other pancreatic disorders (eg chronic pancreatitis, pancreatic mass or cyst, and previous pancreatic surgery or trauma), pregnancy, known case of malignancy, illicit drug abusers, and non-cooperative patients. 'Metabolic syndrome was defined based on National Cholesterol Education Program Adult Treatment Panel III (NCEP/ATP-III). Abbreviations: RAC, revised Atlanta classification; BISAP, Bedside Index of Severity in Acute Pancreatitis.

MetS group than in the non-MetS group $(\mathrm{P}=0.001)$. Overall, 3 patients died, two of whom had MetS and all had severe RAC and BISAP 3-5. Comparison of outcome in AP between MetS and non-MetS groups shown in Table 3.

Comparison of frequency of MetS components in patients with AP based on RAC and BISAP score shown in Tables 4-6. Waist circumference, fasting blood sugar, and triglyceride were significantly higher in the severe and moderately severe AP groups than in the mild AP group, according to RAC (Tables 4 and 5). Although, mean of all components of MetS except HDL-C were higher in BISAP of 3-5 group than BISAP of 0-2 group, but, the difference was only significant in waist circumference $(95.58 \pm$ $9.93 \mathrm{~cm}$ in BISAP of $3-5$ group vs $88.28 \pm 9.92 \mathrm{~cm}$ in BISAP of $0-2$ group, $P=0.027$ ). Robust Poisson regression models was performed for estimating the PRs and $95 \%$ CIs for BISAP score by components of metabolic syndrome (Table 6).

\section{Discussion}

This cross-sectional study in patients with AP showed a significant association between the presence of MetS and the increase in severity of AP based on the RAC as well as the BISAP score. The association between MetS components and AP severity based on NCEP/ATPIII criteria was also investigated. Waist circumference, fasting blood sugar, and triglyceride were significantly higher in the severe and moderately severe types of AP group than in the mild AP group, according to RAC (Table 5). Although, mean of all components of MetS except HDL-C were higher in BISAP of 3-5 group than BISAP of 0-2 group, but, the difference was only 
Table I Baseline Demographic and Clinical Characteristics of Acute Pancreatitis in Metabolic Syndrome (MetS) $(n=34)$ and Non-MetS $(n=42)$ Participants

\begin{tabular}{|c|c|c|c|}
\hline Variables & MetS; N (\%) & Non-MetS; N (\%) & $P$ value \\
\hline Gender $^{\mathrm{b}}$ & & & 0.331 \\
\hline Female & $14(4 \mid .2 \%)$ & $22(52.4 \%)$ & \\
\hline Male & $20(58.8 \%)$ & $20(47.6 \%)$ & \\
\hline Age $^{c}$ (years) & $50.59(14.07)$ & $45.71(16.75)$ & 0.180 \\
\hline Waist circumference $(\mathrm{cm})^{\mathrm{d}}$ & $93.97(9.68) / 95$ & $85.76(9.19) / 84.5$ & 0.001 \\
\hline Fasting blood sugar $(\mathrm{mg} / \mathrm{dL})^{\mathrm{d}}$ & $130.00(33.98) / 119$ & $85.52(13.66) / 85$ & $<0.001$ \\
\hline Triglyceride $(\mathrm{mg} / \mathrm{dL})^{\mathrm{d}}$ & $190.29(44.50) / 188$ & I $42.83(27.34) / / 40$ & $<0.001$ \\
\hline High-density lipoprotein $(\mathrm{mg} / \mathrm{dL})^{d}$ & $38.35(8.75) / 35$ & $48.36(8.02) / 50.5$ & $<0.001$ \\
\hline SBP/DBP $\geq 130 / 85 \mathrm{mmHg}^{\mathrm{b}}$ & $16(47.1 \%)$ & II (26.2\%) & 0.059 \\
\hline
\end{tabular}

Notes: a National Cholesterol Education Program Adult Treatment Panel III was used for diagnosis of metabolic syndrome. ${ }^{\mathrm{b}} \mathrm{Chi}-\mathrm{square}$ test. ${ }^{c} t$-Test; mean (standard deviation). ${ }^{d}$ Mann-Whitney test; mean (standard deviation)/median.

Abbreviations: SBP, systolic blood pressure; DBP, diastolic blood pressure.

Table 2 Comparison of Severity of Acute Pancreatitis Between Metabolic Syndrome ${ }^{a}$ (MetS) $(n=34)$ and Non-MetS $(n=42)$ Groups

\begin{tabular}{|l|l|l|l|}
\hline Variables & $\begin{array}{l}\text { MetS; } \\
\text { N (\%) }\end{array}$ & $\begin{array}{l}\text { Non-MetS; } \\
\text { N (\%) }\end{array}$ & P value \\
\hline $\begin{array}{l}\text { BISAP score } \\
0-2\end{array}$ & $25(73.5 \%)$ & $39(92.9 \%)$ & 0.022 \\
$3-5$ & $9(26.5 \%)$ & $3(7.1 \%)$ & \\
\hline BISAP score & & & \\
0 & $10(29.4 \%)$ & $22(52.4 \%)$ & 0.043 \\
I & $9(26.5 \%)$ & $11(26.2 \%)$ & 0.976 \\
2 & $6(17.6 \%)$ & $6(14.3 \%)$ & 0.694 \\
3 & $5(14.7 \%)$ & $3(7.1 \%)$ & 0.282 \\
4 & $3(8.8 \%)$ & $0(0.0 \%)$ & 0.049 \\
5 & $1(2.9 \%)$ & $0(0.0 \%)$ & 0.266 \\
\hline RAC & & & 0.029 \\
Mild & $20(58.8 \%)$ & $36(85.7 \%)$ & \\
Moderately severe & $11(32.4 \%)$ & $5(11.9 \%)$ & \\
Severe & $3(8.8 \%)$ & I (2.4\%) & \\
\hline
\end{tabular}

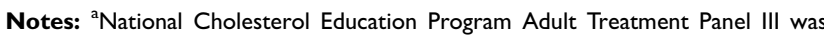
used for diagnosis of metabolic syndrome. ${ }^{\text {b} C h i-s q u a r e ~ t e s t . ~}$

Abbreviations: BISAP, Bedside Index of Severity in Acute Pancreatitis; RAC, revised Atlanta classification.

significant in waist circumference (Table 6). Also, the frequency of complications and length of stay in MetS group was significantly higher than non-MetS group (Table 3).

AP is a common clinical problem with high costs of treatment. AP has many causes, of which alcohol and gallstones account for $70 \%$ in adults. In children, etiologies are broader and more variable. ${ }^{1}$ AP begins with pancreatic inflammation, which can lead to peripancreatic or multi-organ involvement. Most cases of acute pancreatitis are mild and self-limiting and have no sequelae, but
Table 3 Comparison of Outcome in Acute Pancreatitis Between Metabolic Syndrome (MetS) $(n=34)$ and Non-MetS $(n=42)$ Groups

\begin{tabular}{|c|c|c|c|}
\hline Outcome & $\begin{array}{l}\text { MetS; } \\
\text { N (\%) }\end{array}$ & $\begin{array}{l}\text { Non-MetS; } \\
\text { N (\%) }\end{array}$ & $P$ value \\
\hline Complications $^{\mathrm{a}, \mathrm{b}}$ & 14 (4I. $2 \%)$ & $6(14.3 \%)$ & 0.008 \\
\hline $\begin{array}{l}\text { Duration of } \\
\text { hospitalization (days) }\end{array}$ & $6.88(3.98)$ & $4.21(1.84)$ & 0.001 \\
\hline Mortality ${ }^{b}$ & $2(5.9 \%)$ & I (2.4\%) & 0.436 \\
\hline
\end{tabular}

Notes: aSystemic and local complications including fluid collection, pseudocyst, necrotizing pancreatitis, organ failure, and biliary obstruction. ${ }^{\mathrm{b}} \mathrm{Chi}$-square test. ${ }^{c} t$-Test; mean (standard deviation).

complications and severe illness occurs in some cases. Severe AP has high mortality and its early detection is particularly important for proper management. ${ }^{2}$

A variety of scoring systems are available for the severity and prognosis of AP. BISAP is a simple scoring system that is performed on admission of AP and predicts the severity of disease. BISAP scores are used within 24 hours of hospitalization for AP patients who are at risk for severe illness, organ failure and possibly dying in hospital because BISAP can very well predict organ failure and death in the disease. ${ }^{3-5}$ The RAC is another standard clinical and radiological classification to estimate the severity of AP. Organ failure and local or systemic complications are used for this type of classification. $^{7-9}$ In our study, all patients with mortality were in the severe RAC group. As mentioned earlier, one of the severity scoring systems is the BISAP score, which can detect patients at risk of death or severe illness early in the AP course. The BISAP score $\geq 3$ appeared to be reliable for identifying patients with high risk AP. Patients with a BISAP 
Table 4 Comparison of Frequency of Metabolic Syndrome ${ }^{a}$ (MetS) Components in Patients with Acute Pancreatitis Based on Revised Atlanta Classification (RAC) $(n=76)$

\begin{tabular}{|l|l|l|l|l|}
\hline MetS Components & Mild RAC & Moderately Severe RAC & Severe RAC & P value \\
\hline Waist circumference $(\mathrm{cm})^{\mathrm{b}}$ & $88.05(10.45)$ & $91.06(8.06)$ & $102.25(3.59)$ & 0.027 \\
${\text { Fasting blood sugar }(\mathrm{mg} / \mathrm{dL})^{\mathrm{b}}}^{\text {Triglyceride }(\mathrm{mg} / \mathrm{dL})^{\mathrm{b}}}$ & $101.52(32.6 \mathrm{I})$ & $108.44(20.85)$ & $148.00(56.79)$ & 0.043 \\
High-density lipoprotein cholesterol (mg/dL) $^{\mathrm{b}}$ & $156.96(42.86)$ & $178.94(32.45)$ & $204.00(54.11)$ & 0.010 \\
SBP/DBP $\geq 130 / 85 \mathrm{mmHg}^{\mathrm{c}}$ & $19(33.9 \%)$ & $5(31.3 \%)$ & $35.50(5.45)$ & 0.137 \\
\hline
\end{tabular}

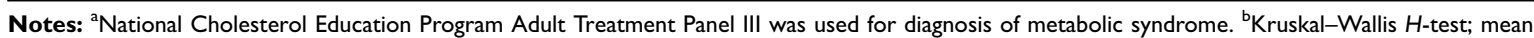
(standard deviation). ${ }^{\mathrm{C}} \mathrm{Chi}$-square test.

Abbreviations: SBP, systolic blood pressure; DBP, diastolic blood pressure.

Table 5 Comparison of Metabolic Syndrome ${ }^{a}$ Components in Mild Patients versus Other Acute Pancreatitis Groups Based on Revised Atlanta Classification (RAC) $(n=76)$

\begin{tabular}{|c|c|c|c|}
\hline Metabolic Syndrome Components & Mild RAC & Severe and Moderately Severe RAC & $P$ value \\
\hline Waist circumference $(\mathrm{cm})^{\mathrm{b}}$ & $88.05(10.45)$ & $93.30(8.63)$ & 0.028 \\
\hline Fasting blood sugar $(\mathrm{mg} / \mathrm{dL})^{b}$ & $101.52(32.6 \mathrm{I})$ & $116.35(33.40)$ & 0.026 \\
\hline Triglyceride $(\mathrm{mg} / \mathrm{dL})^{\mathrm{b}}$ & $156.96(42.86)$ & $183.95(37.40)$ & 0.003 \\
\hline High-density lipoprotein cholesterol (mg/dL) ${ }^{\mathrm{b}}$ & $44.63(10.00)$ & $41.80(8.66)$ & 0.137 \\
\hline SBP/DBP $\geq 130 / 85 \mathrm{mmHg}^{\mathrm{c}}$ & $19(33.9 \%)$ & $8(40.0 \%)$ & 0.626 \\
\hline
\end{tabular}

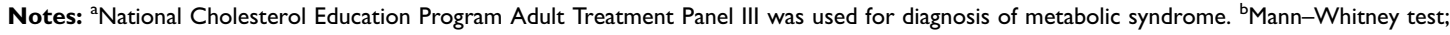
mean (standard deviation). ${ }^{\mathrm{C}} \mathrm{Chi}$-square test.

Abbreviations: SBP, systolic blood pressure; DBP, diastolic blood pressure.

score $\geq 3$ significantly has a higher mortality rates. ${ }^{6}$ In our study, all patients with mortality had a BISAP score of 3-5.

MetS as a common clinical problem has been identified as a combination of several metabolic abnormalities and is associated with increased mortality and morbidity. ${ }^{10,11}$ The relationship between MetS and severity of AP is unclear, and there is a paucity of literature about this issue. ${ }^{12}$ Sawalhi et al evaluated 140 patients with AP to assess the impact of obesity and metabolic syndrome based on International Diabetic Federation definition on the AP course. $88.6 \%$ and $11.4 \%$ of their patients had mild and severe AP, respectively, that $62.8 \%$ of patients with AP had MetS. They concluded that the presence of MetS did not affect the severity of AP, whereas obesity correlated with the severity of AP. ${ }^{14}$ Compared with this study, in addition to waist circumference, fasting blood glucose and triglycerides were significantly higher in the severe and moderately severe AP groups than the mild group, according to the RAC. On the other hand, the mean of all components of MetS except HDL-C were higher in BISAP of 3-5 group than BISAP of 0-2 group, but, the difference was only significant in waist circumference.

Table 6 Robust Poisson Regression Models Estimating PR and 95\% Cl for BISAP Score by Components of Metabolic Syndrome ${ }^{\mathrm{a}}$

\begin{tabular}{|c|c|c|c|c|}
\hline \multirow[t]{2}{*}{ Components of Metabolic Syndrome } & \multicolumn{2}{|l|}{ Crude Model } & \multicolumn{2}{|l|}{ Adjusted Model } \\
\hline & PR (95\% Cl) & $P$ value & PR (95\% Cl) & P value \\
\hline Waist circumference $(\mathrm{cm})$ & $1.008(1.001-1.015)$ & 0.019 & $1.008(1.001-1.015)$ & 0.019 \\
\hline Fasting blood sugar $(\mathrm{mg} / \mathrm{dL})$ & $1.002(1.000-1.004)$ & 0.120 & $1.001(0.998-1.003)$ & 0.511 \\
\hline Triglyceride (mg/dL) & $1.001(1.000-1.003)$ & 0.156 & $1.000(0.999-1.002)$ & 0.795 \\
\hline High-density lipoprotein cholesterol $(\mathrm{mg} / \mathrm{dL})$ & $0.997(0.990-1.004)$ & 0.420 & $1.002(0.993-1.010)$ & 0.725 \\
\hline SBP/DBP $\geq 130 / 85 \mathrm{mmHg}$ & & 0.086 & & 0.134 \\
\hline No & $0.875(0.752-1.019)$ & & $0.894(0.773-1.035)$ & \\
\hline Yes & 1.0 & & 1.0 & \\
\hline
\end{tabular}

Note: ${ }^{a}$ National Cholesterol Education Program Adult Treatment Panel III was used for diagnosis of metabolic syndrome.

Abbreviations: BISAP, Bedside Index of Severity in Acute Pancreatitis; SBP, systolic blood pressure; DBP, diastolic blood pressure; PR, prevalence ratio; Cl, confidence interval. 
A study of 690 patients with AP by Mikolasevic et al has been conducted to investigate the effect of MetS on AP severity based on revised Atlanta classification, complications, and survival rates. In this study, similar to our study, the severity of the disease in the MetS group was statistically higher than the non-MetS group. They also found that MetS patients had a significantly higher mortality rate than non-MetS patients (16\% vs $4.5 \%)$. Mortality in our patients was also higher in MetS patients $(5.9 \%)$ than in non-MetS patients $(2.4 \%)$, but the difference was not statistically significant. They also showed that patients with MetS had statistically significant local and systemic complications, which was consistent with our results. The duration of hospitalization did not differ between the MetS and non-MetS groups, but in our study the length of hospital stay was higher in group MetS than in group non-MetS, which was statistically significant. ${ }^{12}$

In a study by Szentesi et al, they concluded obesity, hypertension, and hyperlipidemia were independent risk factors for a number of complications but only hypertension was an independent risk factor for severity of AP. In our study, though, the complications of AP were significantly higher in the MetS group than in the non-MetS group, but unlike the study mentioned, hypertension was not associated with an increase in AP severity based on the RAC and BISAP score. ${ }^{17}$

In our study, increasing waist circumference, as an important indicator of obesity, was statistically significant associated with AP severity based on both criteria of the RAC and BISAP score (Tables 4-6). This result is consistent with the results of most studies suggesting that obesity has a negative impact on the AP process. ${ }^{12,13}$ O'Leary et al conducted a study aimed to investigate the relationship between the AP severity and abdominal fat distribution measured on computed tomography scan. They concluded a strong association between visceral fat and severe AP and complications. ${ }^{18}$ In a scoping study by Goodger et al, they showed that obesity plays an important role in worse outcomes in AP, including an increased risk of severe AP that was consistent with our research. They recommended that further research be conducted on the effect of MetS on outcomes in AP. ${ }^{15}$

This is the first report to show the association between MetS in severity of AP from Iran. Although our study is limited by its small sample size, the main strength of our research was the thorough evaluation of the MetS components, severity of AP, and optimal follow-up of all included patients. Another strength of our study was the simultaneous evaluation of AP severity based on the two criteria of RAC and BISAP in all participants for better comparison.

\section{Conclusions}

The present study found a significant association between MetS and increased severity of AP. This study was also showed a significant association between increasing of waist circumference, hyperglycemia, and hypertriglyceridemia with risk of severe AP. Although the small sample size of our study makes it difficult to make extensive recommendations, this study showed that the incidence of more severe AP in patients with MetS is much higher than in patients without MetS. However, the mechanism of these associations is still unknown, so further research is needed to clarify the relationship between the nature of MetS and AP.

\section{Acknowledgment}

This study was a part of a MD thesis written by Jamshid Moradi and was supported by the Research Council of Shiraz University of Medical Sciences, Shiraz, Iran (9501-01-11662).

\section{Disclosure}

The authors report no conflicts of interest in this work.

\section{References}

1. Guda NM, Muddana V, Whitcomb DC, et al. Recurrent acute pancreatitis: international state-of-the-science conference with recommendations. Pancreas. 2018;47(6):653-666. doi:10.1097/MPA.0000000000001053

2. Lankisch PG, Apte M, Banks PA. Acute pancreatitis. Lancet. 2015;386 (9988):85-96. doi:10.1016/S0140-6736(14)60649-8

3. Hagjer S, Kumar N. Evaluation of the BISAP scoring system in prognostication of acute pancreatitis - A prospective observational study. Int J Surg. 2018;54(Pt A):76-81. doi:10.1016/j.ijsu.2018.04.026

4. Wu BU, Johannes RS, Sun X, Tabak Y, Conwell DL, Banks PA. The early prediction of mortality in acute pancreatitis: a large population-based study. Gut. 2008;57(12):1698-1703. doi:10.1136/ gut.2008.152702

5. Harshit Kumar A, Singh Griwan M. A comparison of APACHE II, BISAP, Ranson's score and modified CTSI in predicting the severity of acute pancreatitis based on the 2012 revised Atlanta classification. Gastroenterol Rep (Oxf). 2018;6(2):127-131. doi:10.1093/gastro/ gox029

6. Gao W, Yang HX, Ma CE. The value of BISAP score for predicting mortality and severity in acute pancreatitis: a systematic review and meta-analysis. PLoS One. 2015;10(6):e0130412. doi:10.1371/journal. pone. 0130412

7. Banks PA, Bollen TL, Dervenis C, et al. Classification of acute pancreatitis-2012: revision of the Atlanta classification and definitions by international consensus. Gut. 2013;62(1):102-111. doi:10.1136/ gutjnl-2012-302779

8. Foster BR, Jensen KK, Bakis G, Shaaban AM, Coakley FV. Revised Atlanta classification for acute pancreatitis: a pictorial essay. Radiographics. 2016;36(3):675-687. doi:10.1148/rg.2016150097 
9. Colvin SD, Smith EN, Morgan DE, Porter KK. Acute pancreatitis: an update on the revised Atlanta classification. Abdom Radiol (NY). 2019. doi:10.1007/s00261-019-02214-w

10. Grundy SM. Metabolic syndrome update. Trends Cardiovasc Med. 2016;26(4):364-373. doi:10.1016/j.tcm.2015.10.004

11. Zainuddin LR, Isa N, Muda WM, Mohamed HJ. The prevalence of metabolic syndrome according to various definitions and hypertriglyceridemic-waist in malaysian adults. Int J Prev Med. 2011;2(4):229-237.

12. Mikolasevic I, Milic S, Orlic L, et al. Metabolic syndrome and acute pancreatitis. Eur J Intern Med. 2016;32:79-83. doi:10.1016/j.ejim.20 16.04 .004

13. Abu Hilal M, Armstrong T. The impact of obesity on the course and outcome of acute pancreatitis. Obes Surg. 2008;18(3):326-328. doi:10.1007/s11695-007-9298-5

14. Sawalhi S, Al-Maramhy H, Abdelrahman AI, Allah SE, Al-Jubori S. Does the presence of obesity and/or metabolic syndrome affect the course of acute pancreatitis?: a prospective study. Pancreas. 2014;43 (4):565-570. doi:10.1097/MPA.0000000000000028
15. Goodger RL, Asrani VM, Windsor JA, Petrov MS. Impact of metabolic comorbidities on outcomes of patients with acute pancreatitis: a scoping review. Panminerva Med. 2016;58(1):86-93.

16. Expert Panel on Detection E, Treatment of High Blood Cholesterol in A. Executive summary of the third report of the National Cholesterol Education Program (NCEP) expert panel on detection, evaluation, and treatment of high blood cholesterol in adults (Adult Treatment Panel III). JAMA. 2001;285(19):2486-2497. doi:10.1001/jama.285. 19.2486

17. Szentesi A, Parniczky A, Vincze A, et al. Multiple hits in acute pancreatitis: components of metabolic syndrome synergize each other's deteriorating effects. Front Physiol. 2019;10:1202. doi:10.33 89/fphys.2019.01202

18. O’Leary DP, O’Neill D, McLaughlin P, et al. Effects of abdominal fat distribution parameters on severity of acute pancreatitis. World J Surg. 2012;36(7):1679-1685. doi:10.1007/s00268-011-1414-y

Diabetes, Metabolic Syndrome and Obesity: Targets and Therapy

\section{Publish your work in this journal}

Diabetes, Metabolic Syndrome and Obesity: Targets and Therapy is an international, peer-reviewed open-access journal committed to the rapid publication of the latest laboratory and clinical findings in the fields of diabetes, metabolic syndrome and obesity research. Original research, review, case reports, hypothesis formation, expert opinion and commentaries are all considered for publication. The manuscript management system is completely online and includes a very quick and fair peer-review system, which is all easy to use. Visit http://www.dovepress.com/testimonials.php to read real quotes from published authors.

Submit your manuscript here: https://www.dovepress.com/diabetes-metabolic-syndrome-and-obesity-targets-and-therapy-journal 